Bukh, JetTe

\title{
The Village Woman in Ghana
}

Uppsala 1979, 118 S. Centre for Development Research, Kopenhagen, Bd. 1. (Publisher: Scandinavien Institute of African Studies)

Bukh hat in mehreren Aufenthalten in Südostghana in einem Dorf des patrilinear organisierten Ewe-Stammes eine empirische Studie durchgeführt. Erst bei ihren letzten Aufenthalten ist sie der Frage der Arbeitsteilung der Geschlechter, des Rollenwandels der Frau in der Dorfgesellschaft etc. nachgegangen, wodurch ein Vergleich mit der ersten Untersuchungsphase nur bedingt möglich ist.

Die Studie ist eine interessante Ergänzung zu der bereits recht umfangreichen Frauenliteratur Westafrikas, die wegen der dort vorherrschenden starken Position der Frauen seit langem in Fachkreisen umfassend diskutiert wurde.

Die soziale und wirtschaftliche Entwicklung seit der Jahrhundertwende, die durch die Einführung des Kakaoanbaues bewirkt wurde (Kap. 2), ist die interessanteste Darstellung der Studie. In den übrigen Kapiteln (vor allem Kap. 4 und 5) werden Detailinformationen über die heutige Tätigkeit der Ewe-Frauen gegeben, die vor allem für diejenigen von Interesse sind, die in der ländlichen Beratung tätig sind.

Allgemein zu beobachtende Entwicklungen, wie das steigende Desinteresse der Landbevölkerung an der jetzigen Ausbildungsform, wie die Land-Stadt- und Stadt-Land-Migration werden auch hier wieder bestätigt.

Die Stärke der Studie liegt vor allem darin, daß durch die Entwicklung gezeigt wird, daß Frauen nicht überall in Westafrika und seit jeher für die Nahrungsproduktion auf Subsistenzebene verantwortlich waren, sondern daß dies - wie das Beispiel der Ewe zeigt erst mit der Einführung der Kakaoproduktion erfolgte. Bis zu dieser Zeit waren Männer für die Nahrungsmittelproduktion verantwortlich.

Die Einführung der Verkaufsfrucht Kakao hat das bis dahin ausgewogene Arbeitsverhältnis von Mann und Frau (z. B. gemeinsames Arbeiten auf dem Feld) grundlegend verändert. $\mathrm{Da}$ in dem partiarchalischen System die Geldwirtschaft ausschließlich in der Hand der Männer war, schufen sich Frauen Arbeitsbereiche, die ihnen ein eigenes Einkommen sicherstellten. Hier ist vor allem der Handel mit selbsthergestellter Ware und die Bewirtschaftung von Land, das nicht dem männlichen Familienstand gehört, zu nennen.

In den letzten Jahren ist eine Lockerung des Familienverbandes festzustellen, was vor allem darauf zurückzuführen ist, daß der Einfluß der Alten vermindert worden ist. Heiraten werden kaum noch durch die Eltern arrangiert, die Entscheidung treffen die Heiratswilligen selbst. Durch die wirtschaftliche Eigenständigkeit der Frau ist diese nicht mehr gezwungen, alle Mißstände zu ertragen. Dies macht sich vor allem in der hohen Scheidungsrate (2/3) bemerkbar, wobei zumeist die Frau den Antrag auf Trennung stellt.

Neben den wirtschaftlichen und familiären Auswirkungen hat die Einführung des Kakaoanbaues auch ernährungsphysiologische Veränderungen bewirkt. Statt der arbeitsintensiven, aber inhaltsreichen Anbaufrucht Yams mit dem dazwischen anbaubaren Gemüse wird Cassava von den Frauen angebaut. Da sich die Männer auf den Anbau der Verkaufsfrucht Kakao konzentrieren, steht ihre Arbeitskraft für den zeitaufwendigen Yamsanbau nicht mehr zur Verfügung, so daß die Frauen zur Deckung des Grundnahrungsmittelbedarfs auf Cassava ausweichen müssen, ohne daß die höheren Bareinnahmen der Familie zur Kompensierung der minderwertigen Cassava-Ernährung genutzt würden, so daß sich die Ernährungslage der Bevölkerung verschlechtert hat. 
Erste positive Ansätze der Selbsthilfe der Frauen (Sparvereine, Frauenorganisation etc.) sind vorhanden, jedoch noch zu neu, um die Möglichkeit für einen längerfristigen Wandel der Arbeitsbelastung der Frauen beurteilen zu können.

Da die Studie sich weitgehend nur auf das eine Untersuchungsdorf bezieht, kann nicht gesagt werden, ob die Aussagen für den gesamten Stamm der Ewe Gültigkeit hat. Ein Umstand, der leider die Aussagefähigkeit der ansonsten interessanten Darstellung einschränkt.

Gudrun Martius-von Harder

Leo E. Rose

\section{The Politics of Bhutan}

Cornell University Press, Ithaca, London, 1977, 237 S., \$ 19,50

Der Himalaya-Staat Bhutan, an Fläche wenig größer als die Schweiz, hat ca. 12 Millionen Einwohner, davon $75 \%$ Buddhisten und $20 \%$ Hindus. Bis Anfang der sechziger Jahre verfügte er nicht über moderne Straßen, noch heute ist er per Flugzeug nur von Indien aus zu erreichen. Im Schrifttum wird Bhutan gern als ,, Museum einer asiatischen Hochkultur“ bezeichnet, ansonsten auf seine Armut, die Analphabetenrate von $95 \%$ und die Abhängigkeit von Indien hingewiesen. Im übrigen entspricht der geographischen und politischen Isolierung des Landes (Bhutan ist zwar seit 1971 Mitglied der Vereinten Nationen, unterhält aber beispielsweise weder diplomatische Beziehungen zur Bundesrepublik noch zur DDR) eine fast ausnahmslose sozialwissenschaftliche Vernachlässigung.

Dieser Zustand ist aus mehreren Gründen bedauerlich. Der Wissenschaft von den internationalen Beziehungen bietet die Geschichte Bhutans, des Pufferstaates zwischen zwei ganz unterschiedliche außen- und innenpolitische Wege verfolgenden asiatischen Entwicklungsländern, reiches Anschauungsmaterial für die Möglichkeiten eines Kleinstaates, Annexion und Assimilierung vorzubeugen; der völkerrechtlichen Lehre über Protektorat und Kolonien liefern die verschiedenen Abhängigkeitsformen Bhutans von Tibet, China, Großbritannien und Indien Anschauungsmaterial; wer schließlich die Konfrontation einer traditionalen, stark religiös geprägten, hierarchischen (aber auch: von Hunger freien) Gesellschaft der Dritten Welt mit Technologie und ,,modernen“ Werten, die Auswirkungen dessen auf Herrschaftssystem, Verfassungsstruktur, Justizapparat untersucht, findet in Bhutan ein bemerkenswertes Beispiel.

$\mathrm{Zu}$ allen genannten Aspekten liefert die Studie von Leo E. Rose Informationen und überzeugende, sorgfältig begründete Einschätzungen. Der Autor hat sich zwischen 1972 und 1975 mehrmals im Lande aufgehalten und war gezwungen, die wenigen bislang vorliegenden (meist in Indien publizierten) Informationen über Bhutan durch persönliche Interviews zu ergänzen: Nicht nur Meinungen, auch schon neuere Fakten waren nur auf diese Weise zu recherchieren. Rose verknüpft sie mit souveräner Kenntnis von Geschichte und Kultur der Region (er war 1960 bis 1968 Direktor des Himalayan Border Countries Project der Universität in Berkeley und ist Herausgeber des ,, Asian Survey“), zeigt daneben auch Aufgeschlossenheit für neuere entwicklungspolitische und für rechtsvergleichende Fragestellungen. Er zeichnet zunächst die historische Entwicklung Bhutans nach, das im 16. Jahrhundert staatliche Identität erreichte. Es entstand ein theokratisches System, das dem tibetischen stark ähnelte, wie auch schon zuvor tibetische Einflüsse in dem Territorium zumeist gegenüber den indischen überwogen. Tibetische Einwanderer prägten die Entwicklung Bhutans, doch handelte es sich zumeist um politische Flüchtlinge, so daß eine „Kolonisierung“ Bhutans durch Tibet allein dadurch nicht zustande kam. Freilich führten militärische und diplomatische Aktionen Tibets und auch Chinas dazu, daß Bhutan zeitweise deren Einflußgebiet 